\title{
Argyrophilic thorny astrocyte clusters in association with Alzheimer's disease pathology in possible primary progressive aphasia
}

\author{
David G. Munoz $\cdot$ John Woulfe $\cdot$ Andrew Kertesz
}

Received: 29 March 2007 / Revised: 29 June 2007 / Accepted: 29 June 2007 / Published online: 19 July 2007

(C) Springer-Verlag 2007

\begin{abstract}
Although most cases of primary progressive aphasia (PPA) have one of the varieties of frontotemporal lobar degeneration (FTLD) as their pathological substrate, a subset shows Alzheimer's disease (AD) pathology. We report that all eight cases in our clinic diagnosed as possible PPA, on account of the presence of episodic memory difficulties in addition to severe language impairment at the onset of disease, showed AD pathology. Neither focal accentuation of $\mathrm{AD}$ pathology nor vascular lesions in language-related areas was observed. Seven of these eight patients showed large argyrophilic thorny astrocyte clusters (ATAC) in the fronto-temporo-parietal cortex and subcortical white matter. The intensely tau immunoreactive astrocytes in ATAC were morphologically similar to the perivascular, subpial, and subependymal astrocytes in elderly brains, but ATAC differ from the latter by the cortical and subcortical location, widespread distribution outside the medial temporal lobe, and intense argyrophilia. The location of ATAC was related to neither local variations in the load of AD pathology, nor the myelin density of white
\end{abstract}

D. G. Munoz

Li Ka Shing Knowledge Institute of St. Michael's Hospital,

University of Toronto, Toronto, ON, Canada

J. Woulfe

University of Ottawa and The Ottawa Health

Research Institute, Ottawa, ON, Canada

A. Kertesz

University of Western Ontario, London, ON, Canada

D. G. Munoz $(\bowtie)$

Department of Laboratory Medicine and Pathobiology,

St. Michael's Hospital, 30 Bond St, Rm CC 2-097,

M5B 1W8 Toronto, ON, Canada

e-mail: dave_munoz@yahoo.com matter. ATAC were not seen in a comparison group of six cases of $\mathrm{AD}$ without a prominent aphasia syndrome. Because of the similarity of astrocytes in ATAC to those seen independently of $\mathrm{AD}$ pathology in several subtypes of FTLD and two reported cases of PPA we hypothesize that they are a marker of a pathological process concurrent with $\mathrm{AD}$, and related to the focality of the clinical presentation.

Keywords Tau - Frontotemporal dementia $\cdot$ FTLD . Neurodegeneration $\cdot$ Cerebral cortex $\cdot$ Gallyas

\section{Introduction}

Although described in the original papers of Arnold Pick and other early reports of similar conditions, the recognition of primary progressive aphasia (PPA) as a relatively common syndrome followed the seminal work of Marsel Mesulam [45]. He defined PPA as a gradual isolated dissolution of language, contrasting with the preservation of other cognitive functions and behavior [46]. The structural basis of PPA has remained controversial [33].

Neuropathological examination of autopsied cases or of brain biopsies have shown that PPA is most commonly associated with different forms of frontotemporal lobar degeneration (FTLD) [5], including FTLD with Pick bodies $[14,20,31,66]$, corticobasal degeneration (CBD) [9, 22, 39], FTLD with motor neuron disease-type inclusions or ubiquitin-only inclusions (FTLD-U) [34, 60], and dementia lacking distinctive histopathology $[7,15,35,44,57,59$, 61], although the latter diagnosis, always questionable, cannot even be considered in the absence of ubiquitin immunostains.

On the other hand, a number of reported cases of PPA have shown as the only recognized pathology neurofibrillary 
tangles and senile plaques in a density sufficient for the diagnosis of Alzheimer's disease (AD) by current criteria. As many as one third of patients diagnosed clinically as either progressive non-fluent aphasia (PNFA) or semantic dementia showed this pattern [36]. Some reports have remarked the relative sparing of medial temporal lobe structures (hippocampus and entorhinal cortex) [16, 17, 56]. Galton et al. [10] have reported a large series of atypical presentations of pathologically confirmed $\mathrm{AD}$, which included six cases of PPA. Non-fluent, mixed, and fluent aphasia were represented. Although episodic memory deficits were present in most patients, there were exceptions. Thus, neither a clinical profile of patients with PPA and AD pathology, nor a pathological difference between cases with AD pathology with and without PPA symptoms has been reported.

We first encountered an opportunity to make a contribution to the questions above when we classified 13 patients with PPA into a probable and a possible group, depending on whether non-language memory deficits were present or absent at onset. All ten patients with definite PPA showed FTLD pathology (3 FTLD with Pick bodies, 4 CBD, 3 FTLD-U). In contrast all three patients with memory deficits showed AD pathology [52]. However, there was a surprising lack of correspondence between the widespread AD pathology and the localized clinical manifestation. Later, two of us (DM and JW) observed a distinctive type of glial abnormality in the brains of these patients, and we investigated the presence and distribution of this lesion in all available brains with a reliable diagnosis of possible PPA and a group of AD patients without possible PPA.

\section{Patients and methods}

Among the patients examined by one of us (AK) at the Cognitive Neurology Clinic of the University of Western Ontario, 20 who presented with PPA came to autopsy prior to the end of 2002, and the brains were examined by one of the authors. 12 of these received a diagnosis of probable PPA, when aphasia, whether anomic, logopenic, or nonfluent was the only initial symptom. The pathological diagnosis was one of the varieties of FTLD: FTLD with Pick bodies, CBD, or FTLD-U [32, 53]. Eight patients received a diagnosis of possible PPA because the initial history was considered to have a component of memory impairment accompanying language deficits: they are the subject of the present report. All patients were assessed with the Western Aphasia Battery (WAB) [29]. Other tests used included the mini mental state examination (MMSE), and Mattis dementia rating scale (DRS). The frontal behavioral inventory (FBI) was obtained with the main caregiver.
For comparison, we used six patients with a clinical diagnosis of probable Alzheimer's disease by NINCDS criteria [42] in whom review of the clinical history did not reveal any suggestion of PPA, and who received a pathological diagnosis of Alzheimer disease by CERAD criteria [47].

Brains removed at autopsy were weighed, fixed in formalin for 1-2 weeks, and coronally sectioned. Standard blocks were embedded in paraffin, and $8 \mu \mathrm{m}$ sections stained using the H\&E, Luxol Fast Blue, Bielschowsky, and Gallyas techniques [50]. Immunostains were performed on $4 \mu \mathrm{m}$ sections by the avidin-biotin-peroxidase method, using Vector Elite kit and diaminobenzidine (DAB) as previously described [40]. Appropriate positive and negative controls were used in all cases. For detecting pathological tau proteins we used two mouse monoclonal antibodies, one conformation-dependent, tau-2 (Sigma, 1:200), directed against Ala95 through Ala108 of bovine tau, and specifically for the Ser conformation of the amino-terminal portion of human tau [65], and one phosphorylationdependent, AT8 (Pierce Endogen, 1:100), that recognizes tau protein phosphorylated at both ser 202 and thr 205, [12] (Innogenetics, Ghent, Belgium). Other antibodies used include anti GFAP (Dako, 1:500), anti ubiquitin (Dako, 1:50), anti $\alpha$-synuclein (Zymed, 1:50), and anti Chromogranin A (Dako, 1:2,000). Double labeling was carried out by the sequential method, using a brown chromagen (DAB) for the monoclonal tau antibodies and a purple chromagen (VIP substrate, Vector laboratories) for GFAP. Runs omitting each primary antibody were used as controls.

In the initial evaluation all the clinical information was available to the pathologists. In order to confirm the consistency of the finding reported here ten slides showing argyrophilic thorny astrocyte clusters (ATAC) and ten slides from corresponding areas in the $\mathrm{AD}$ controls were masked and given in random order to one of the pathologist (DM) who correctly identified the presence or absence of ATAC in all cases.

\section{Results}

Clinical

The common feature of the history was the salience of language impairment in contrast to the relative preservation of activities of daily living, navigation, calculation, and planning, even if episodic memory was definitely involved from the outset. For example, a 75-year-old woman was able to drive her car around town without difficulty, depositing or withdrawing money from the bank, maintaining her house, cooking and baking at a time when her language was reduced to garbled speech. The onset was insidious and 
progression gradual in all cases. On examination patients were attentive and cooperative. Word finding difficulty was more apparent on connected speech than on naming. Frequent paraphasias included mostly word substitutions. Impairment of both comprehension and repetition initially involved complex sentences and eventually short ones as well. The following written response to a question about the weather demonstrates the same severe semantic jargon and paragrammatism found in spontaneous speech: "They must dry sometimes wet in (town) in snow after in the monthly". Spontaneous speech fluency varied among patients, depending on the stage they were examined, but characteristically fluency was preserved late into the illness in contrast to PNFA. Constructional apraxia was common, but limb apraxia was not a feature. Preservation of motor skills was exemplified in patients taking long bicycle rides. Many patients developed behavioral symptoms. Some of these such as the mirror and Capgras delusion are in our experience more characteristic of Alzheimer's than Frontotemporal Disease $($ mirror $=$ confusion of a mirror image as a stranger, Capgras $=$ delusion of a close relative as an impostor). The FBI scores averaged 18 (below frontotemporal dementia cutoff, but compatible with PPA or AD).

Eventually patients progressed to a severe global dementia and some to mutism. The clinical features of the eight patients are summarized in Table 1. The WAB scores (for quantization of language features) in the table are from the initial examination. The pattern suggested fluent aphasia ranging from anomia to Wernicke's aphasia, more characteristic of AD than PPA $[1,30]$. Other cognitive test averages/possible maximum: MMSE 8.5/30, DRS 72.8/144 are compatible with other PPA or severe AD patients. The impaired language skills are the likely cause of the low MMSE scores.

Structural brain imaging with CT or MRI showed mild diffuse atrophy in four patients and focal atrophy accompanied by distinct asymmetry in the other four (Fig. 1). In most cases HMPAO-SPECT showed parietal hypoperfusion.

Pathology

Gross exam of the brain in all cases showed diffuse, moderately severe cortical atrophy lacking any obvious lobar accentuation, but often with perisylvian predominance (Fig. 2a), and on serial sections ventricular dilatation and severe hippocampal atrophy (Fig. 2b). In only one case was the atrophy considered grossly asymmetrical, left predominant. On microscopic examination using either Gallyas (Fig. 3a) or tau immunostains (Fig. 3b), all cases showed sufficient neurofibrillary tangles and neuritic plaques to be assigned to the "high likelihood that the dementia was due to AD" category by the NIA-Reagan criteria [21]. The

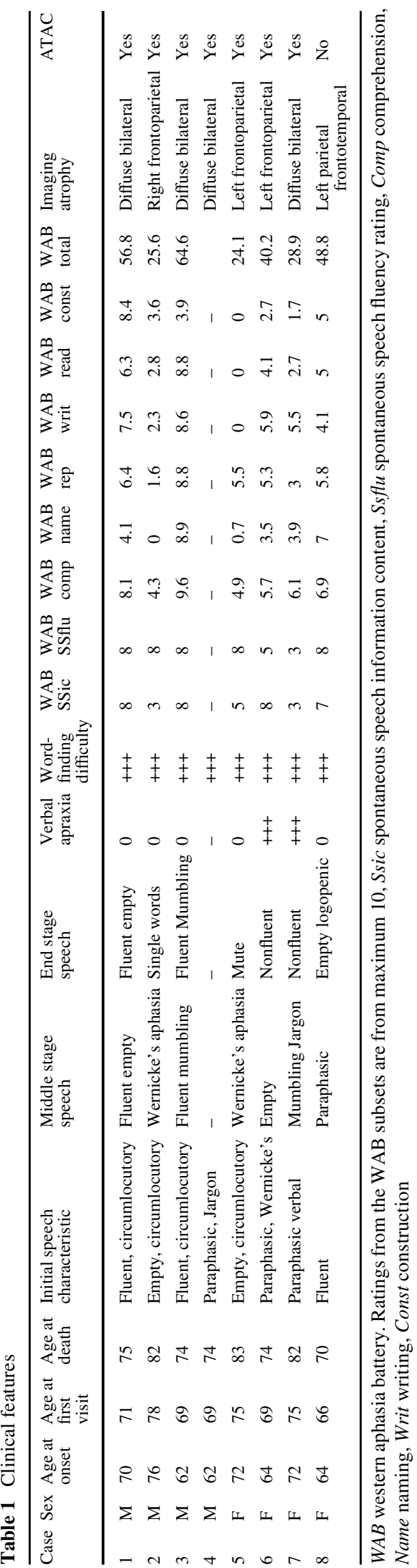


Fig. 1 Magnetic resonance images of patients at presentation. a Patient 7, 74-year-old female, $\mathrm{T} 1$ weighted image, showing distinctly asymmetric atrophy preferentially involving the left cerebral hemisphere. b Patient 1, 75-year-old male, T2 weighted image, demonstrating atrophy primarily affecting the left Sylvian region
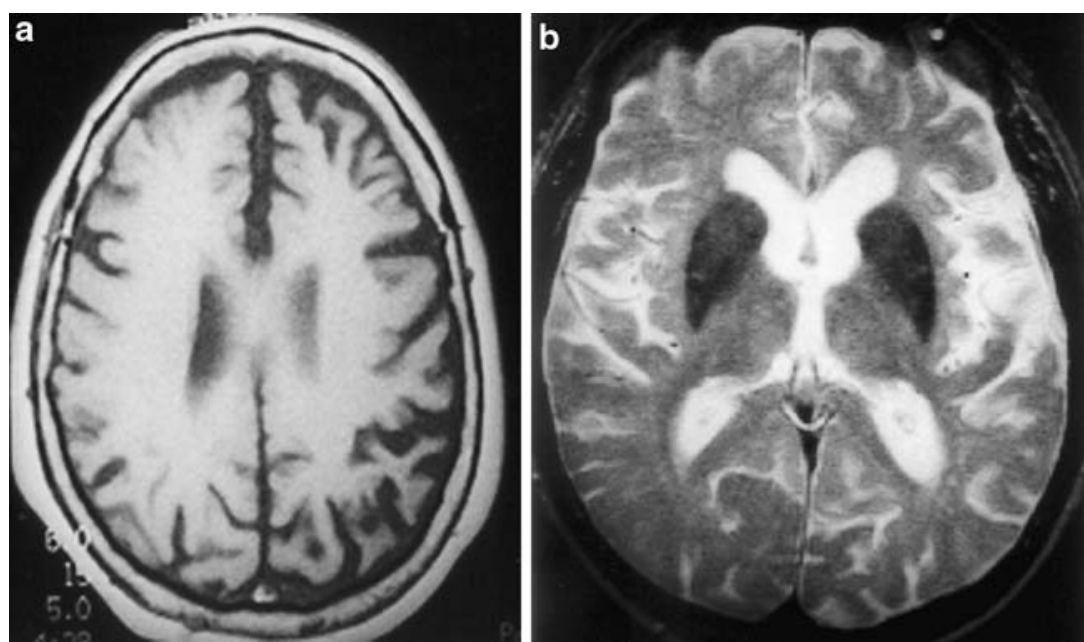
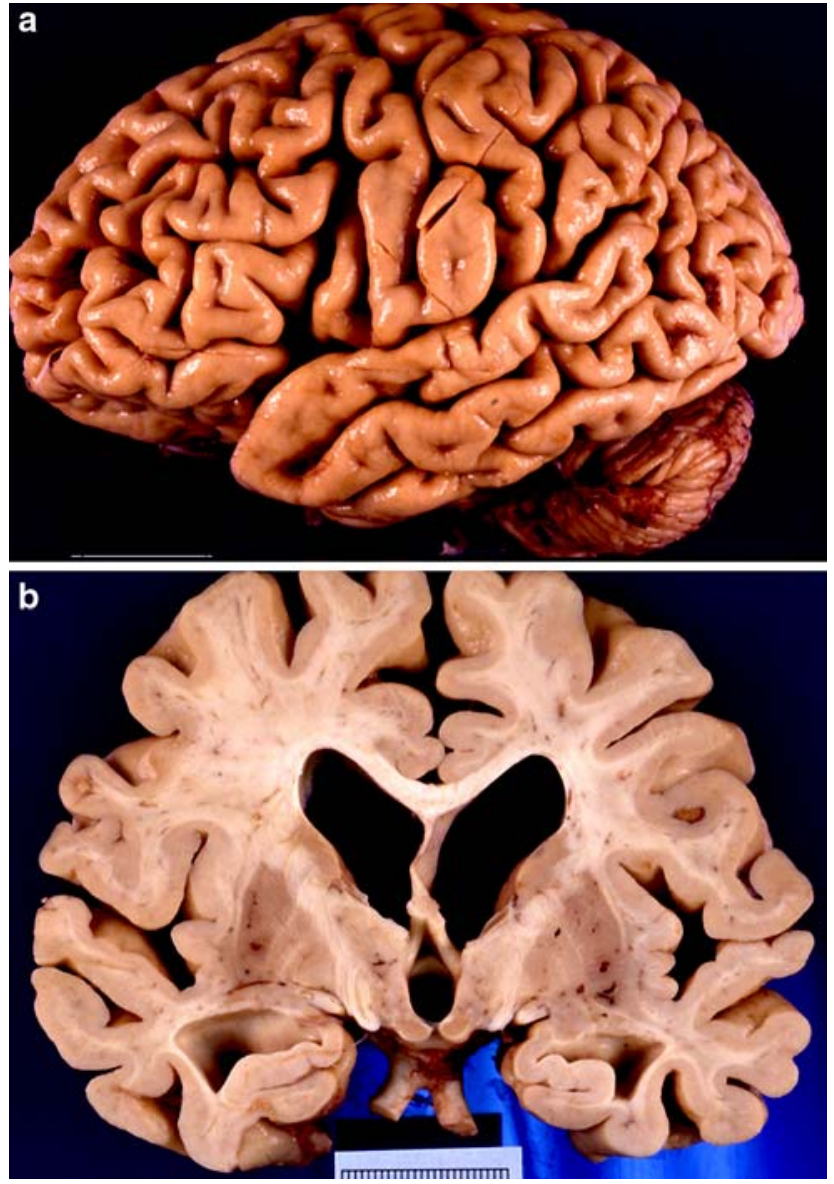

Fig. 2 Gross pathology. a Patient 3, 74-year-old male. Diffuse cortical atrophy lacking any obvious lobar pattern, but with perisylvian accentuation. b Coronal sections demonstrating cortical and hippocampal atrophy with no obvious asymmetry

regional distribution of lesions was consistent with Braak stages 5-6. In one case, a woman aged 70 at the time of death (patient \#8), no additional histological abnormalities
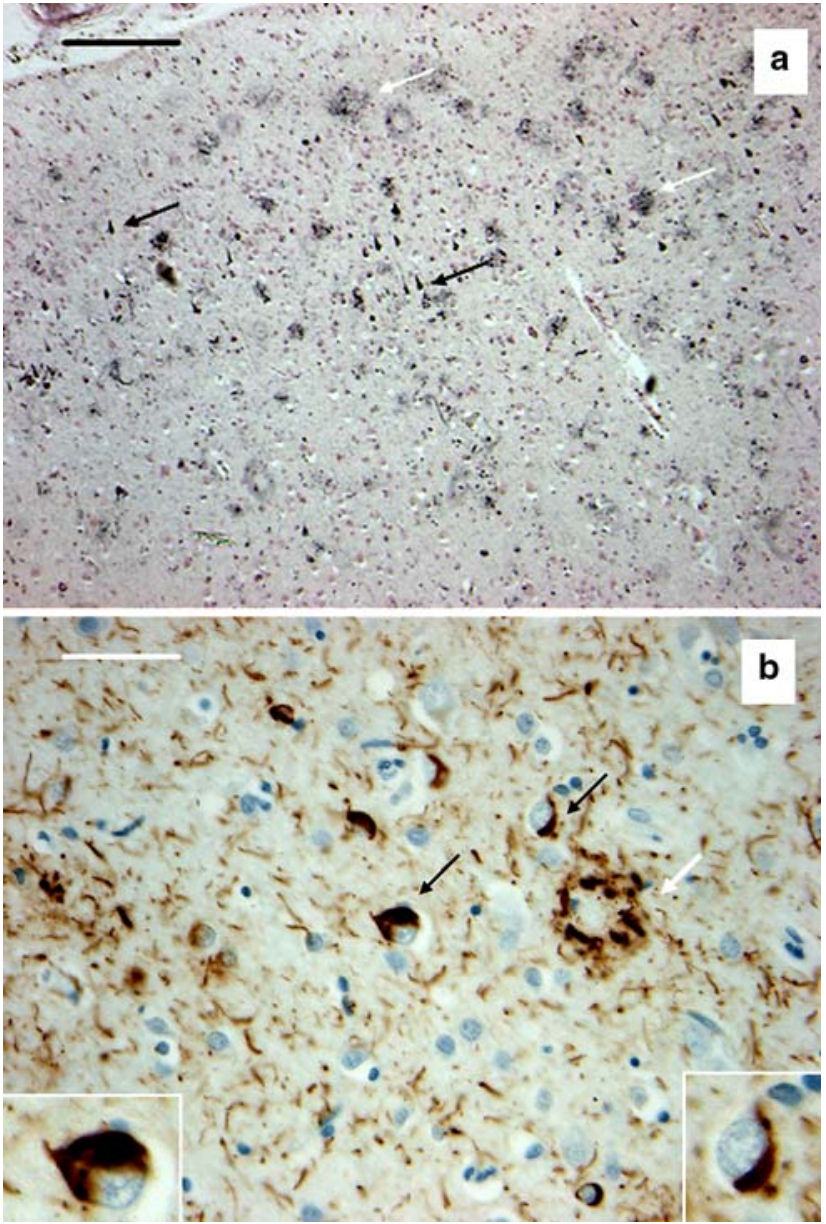

Fig. 3 Alzheimer pathology. The middle frontal gyrus shows neurofibrillary tangles (black arrow) and neuritic plaques (white arrow), utilizing the Gallyas silver stain (a) or tau (AT8) immunostains (b). Two neurofibrillary tangle-bearing neurons are enlarged in the insets. Calibration bars a $200 \mu \mathrm{m}$, b $50 \mu \mathrm{m}$

were present, other than those expected in $\mathrm{AD}$, such as amyloid angiopathy. The other seven cases showed on tau immunostains (Fig. 4) and Gallyas stains (Fig. 5) large 

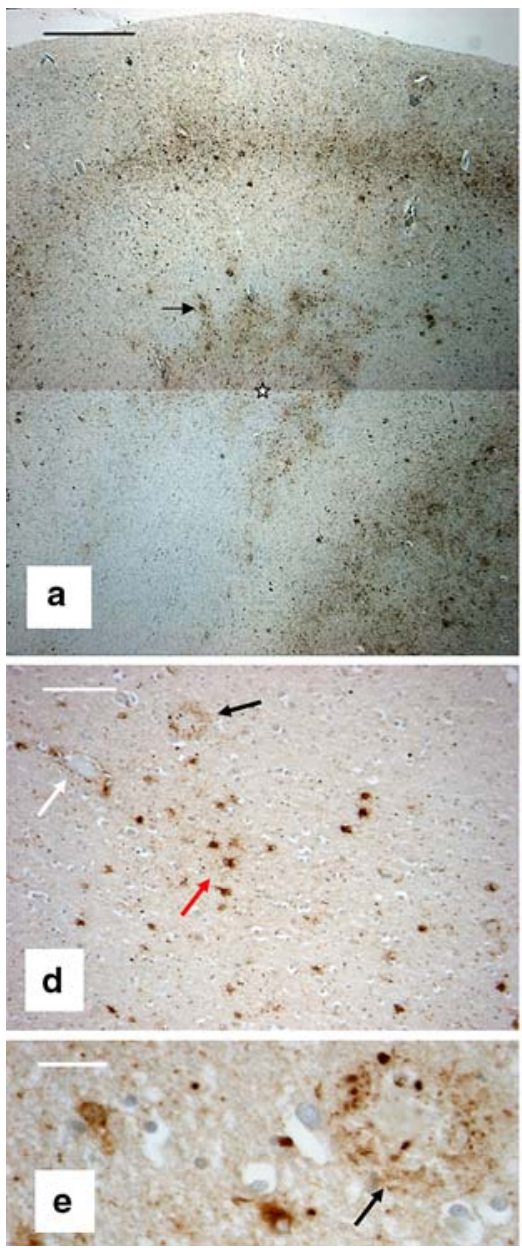

Fig. 4 Argyrophilic thorny astrocyte clusters (ATAC) demonstrated with tau immunostains. a A large ATAC (white asterisk at its center) is present and the subcortical white matter near the grey-white junction. Some extensions form collars around blood vessels (black arrow). Inferior frontal cortex, AT8 immunostain. Bar $1 \mathrm{~mm}$. b The cells in ATAC show a diffuse staining of patchy intensity of stout perikarya with a thorny outline (two labeled with black arrows). Tau-2 immunostain. Bar $20 \mu \mathrm{m}$. c The perivascular collars (asterisk on vessel) are formed by

patchy compact formations in the cortex and subcortical white matter, made up of argyrophilic thorny astrocytes (ATA). We have named these structures ATAC (Figs. 4a, 5a). ATA had an eccentric nucleus and plump perikarya with a thorny outline, the latter labeled by both tau- 2 and AT8 tau antibodies in a patchy pattern of coarse dense strands or clumps, usually on lighter stained background (Fig. 4b). Also stained were a variable proportion of ATA's long varicose processes, often showing sucking feet on reaching the wall of blood vessels (Fig. 4c). ATA did not send processes to neighboring neuritic plaques (Fig. 4d, e). Tau-2 ATA staining was distinctly less intense than neurofibrillary tangles (Fig. 4f), but this was not obvious on AT8 staining. The Gallyas technique detected the same number of clusters and of astrocytes in the clusters as the tau immunostains (Fig. 5a). It stained either the entire perikaryon

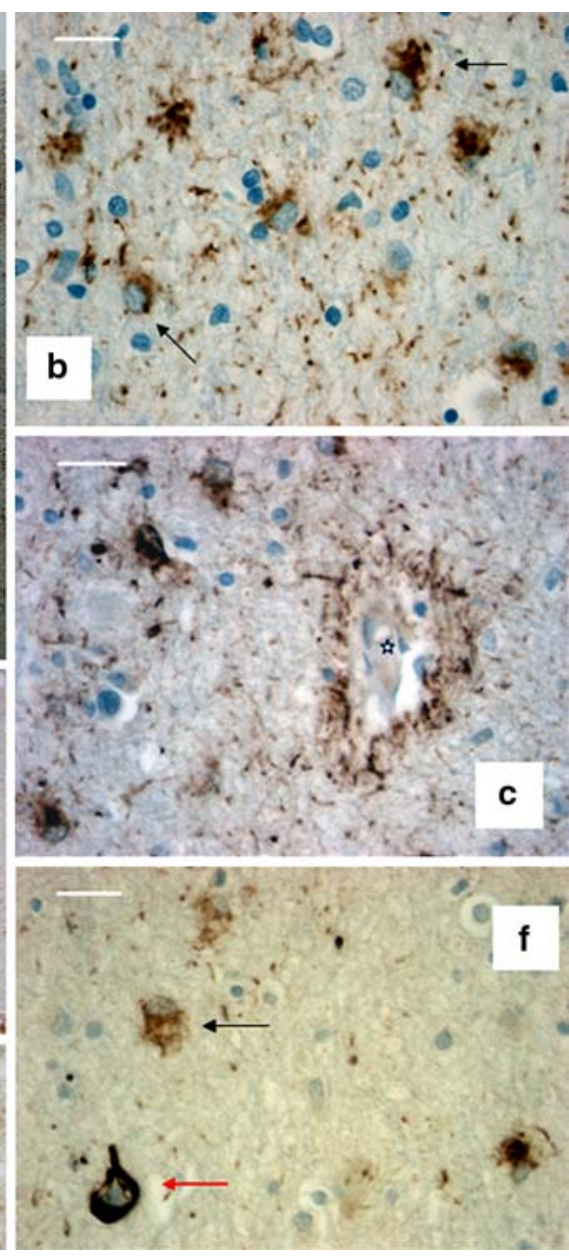

astrocytic processes and their end-feet. AT8 immunostain. Bar $20 \mu \mathrm{m}$. d Unlike the neighboring vessel (white arrow), neuritic plaques in the cortex (black arrow) are not associated with astrocytic process, even if immunolabeled astrocytes (red arrow) are present in the neighborhood. Tau-2 immunostain. Bar $100 \mu \mathrm{m}$. e Higher magnification of d, slightly rotated. The same plaque, with its tau positive neurites, is arrowed. Bar $20 \mu \mathrm{m}$. f Thorny astrocytes (black arrow) are less intensely stained by tau-2 than neurofibrillary tangles (red arrow). Bar $20 \mu \mathrm{m}$

(Fig. 5b) or only some strands (Fig. 5c). The astrocytic nature of the argyrophilic cells was supported by the formation of vascular end-feet (Fig. 5d). When in the cortex ATA were not topographically related to tangle-bearing neurons (Fig. 5e). ATA were not stained by the Bielschowsky technique. Double labeling experiments with GFAP and tau (both tau-2 and AT8) showed some cells intensely expressing both markers, but in most tau-expressing cells GFAP staining is limited to bundles at the perikaryal periphery and the processes (Fig. 6). Astrocyte morphology and areal density was similar regardless of tau expression, and weak or intense GFAP labeling. No immunoreactivity to either ubiquitin or $\alpha$-synuclein was found in ATAC.

The clusters as a whole (ATAC) had a compact oval shape and measured from a few hundred micrometers to $5-10 \mathrm{~mm}$ in diameter. When located in the subcortical white 


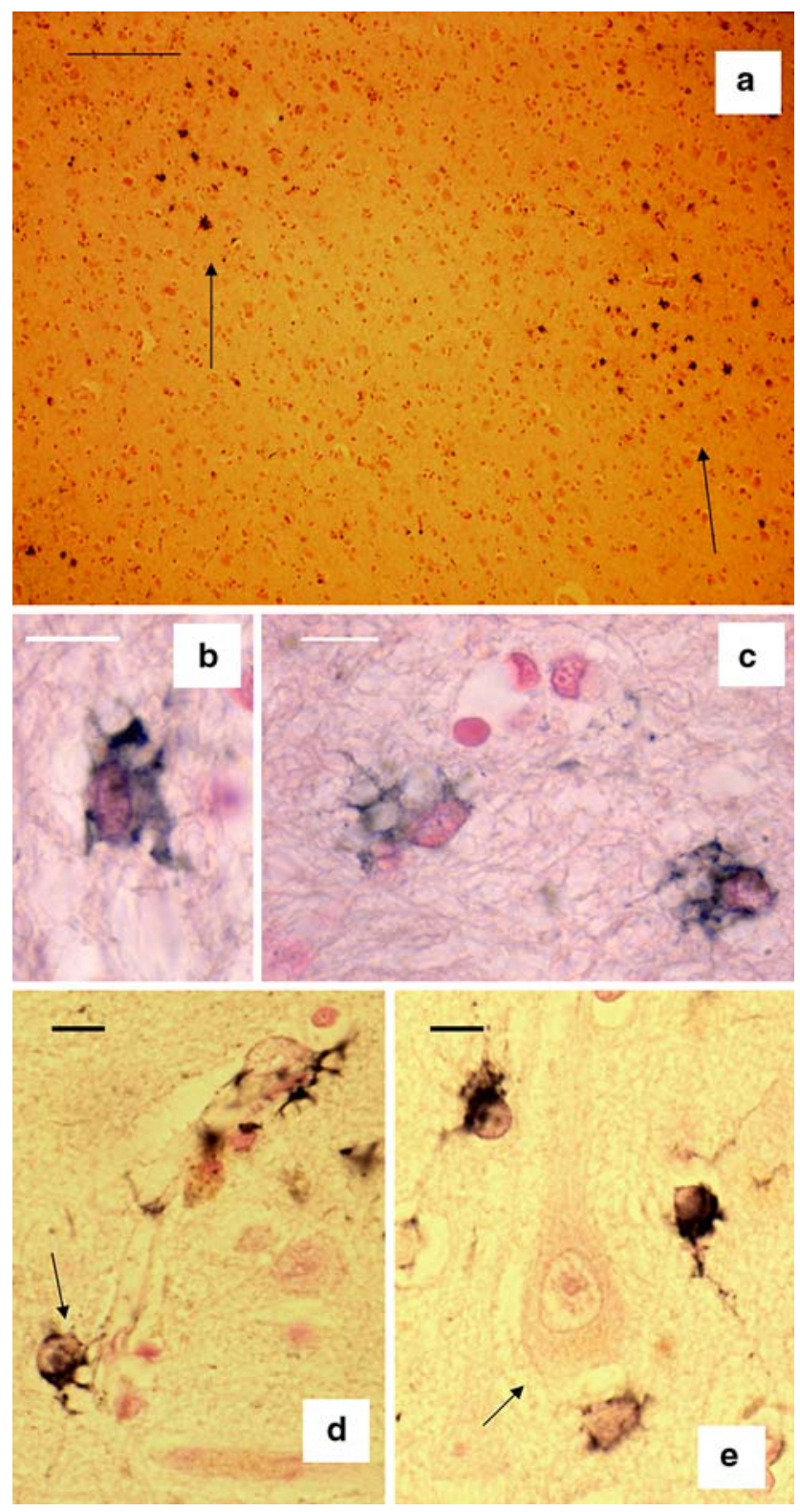

Fig. 5 Argyrophilic thorny astrocyte clusters demonstrated with the Gallyas stain. a Two ATAC (arrows) in the subcortical white matter, frontal lobe. Bar $200 \mu \mathrm{m}$. b A thorny astrocyte with diffuse perikaryal staining, albeit of patchy intensity. Bar $10 \mu \mathrm{m}$. c Staining is limited to strands in the perikarya of these two thorny astrocytes. Bar $10 \mu \mathrm{m}$. d The processes of some thorny astrocytes (arrow) form end-feet on blood vessels. Bar $10 \mu \mathrm{m}$. e Thorny astrocytes in the cortex in the vicinity of a neuron (arrow) lacking neurofibrillary pathology. Bar $10 \mu \mathrm{m}$

matter their margin was parallel to the grey-white junction (Fig. 4a). Blood vessels within an ATAC were surrounded by a halo of argyrophilic, tau immunoreactive processes, but ATAC showed no relationship to subpial or subependymal collections of thorn-shaped astrocytes. White matter areas containing ATAC demonstrated no discernible change on myelin stains, and ATAC could not be delineated on GFAP immunostains in the cortex. ATAC showed no consistent topographic correlation with the density of amyloid deposits or neurofibrillary tangles.

The areas involved were the white and grey matter of the temporal lobe, including the gyrus ambiens, parahippocampal gyrus, fusiform gyrus, inferior, middle, and superior temporal gyri, the frontal dorsolateral and orbitofrontal cortices, the cingulate gyri and occasionally the inferior parietal lobule. They were not found in the insula, occipital lobe, hippocampus, amygdala, thalamus, basal ganglia, brainstem, or cerebellum. There were no obvious focal accentuations of the severity of $\mathrm{AD}$ pathology, or differences between right and left cerebral hemispheres. Neither tufted astrocytes nor glial plaques were seen. Additional neurodegenerative features were the presence of argyrophilic grains and in the parahippocampal gyrus ballooned neurons in a 83-year-old female (patient \#6), and of neurofibrillary tangles in the substantia nigra and periaqueductal grey matter of a 74-year-old male (patient \#3). Severe subicular atrophy with relative sparing of the hippocampus was observed in patients \#3 and \#6. No infarcts were identified on gross examination in any patients, but microscopic infarcts (less than $0.5 \mathrm{~mm}$ in diameter) were observed in the cortex of patients \#1 and \#5, and in the left thalamus of patient \#5.

The group of patients showing ATAC consisted of four men and three women. The average age at onset for men and women was 67.5 and 69.7 years, and at death 76.3 and 79.7, resulting in an average duration until death of 8.8 and 10 years, respectively. Brain weight averaged $1,145 \mathrm{~g}$ for men, and $1,008 \mathrm{~g}$ in women.

None of the six cases of AD without PPA used as the comparison group showed ATAC (Fig. 7).

\section{Discussion}

Although the literature does not describe this association, finding AD pathology as the pathological substrate of possible PPA is not surprising, providing a known basis for the memory impairment accompanying the aphasia. Recently, subtypes of PPA have been identified on clinical, imaging, and genetic bases: PNFA, semantic dementia, and logopenic progressive aphasia. The latter is characterized by anomia, slow speech, and impaired syntactic comprehension clinically, and left posterior temporal cortex and inferior parietal lobule atrophy on imaging. In contrast to the two other forms of PPA, there is over-representation of the ApoE $\varepsilon 4$ allele, as high as $67 \%$, suggesting a link to $\mathrm{AD}$ [13]. The relationship of this form to pathologically identified AD remains to be seen. In the present series the clinical characteristics in the first assessment were closer to the fluent aphasia seen in Alzheimer's disease than the nonfluent aphasia associated with FTLD. Many patients at early 
Fig. 6 Double labeling for GFAP (purple, black arrows) and tau-2 (brown, red arrows). a Most astrocytes within a cluster predominantly express either GFAP or tau. Bar $40 \mu \mathrm{m}$. b-d Co-expression of GFAP and tau is visible in a subset of astrocytes. GFAP may be condensed in bundles at the periphery of the perikaryon (b), in the processes (c), or occupy much of the core of the cell body (d). Bar $10 \mu \mathrm{m}$. e The morphology of processes forming vascular end-feet is similar in astrocytes chiefly expressing either tau or GFAP. Bar $20 \mu \mathrm{m}$. f-g Controls. After omitting the primary antibody for tau, only purple GFAP stained astrocytes are seen (f), whereas omitting the GFAP primary results in brown only staining for tau (g). Bars $\mathbf{f} 40 \mu \mathrm{m}$, g $20 \mu \mathrm{m}$
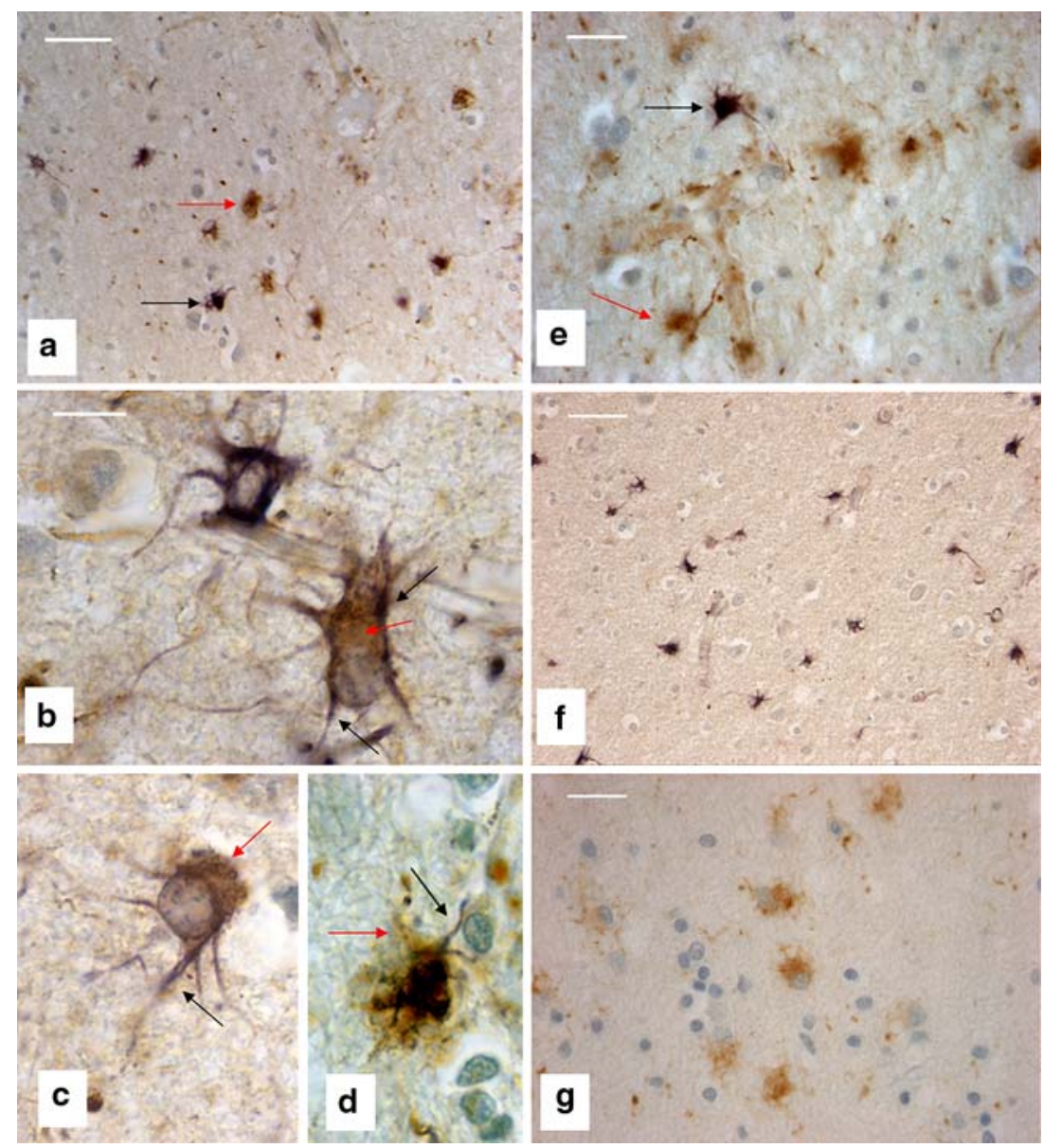

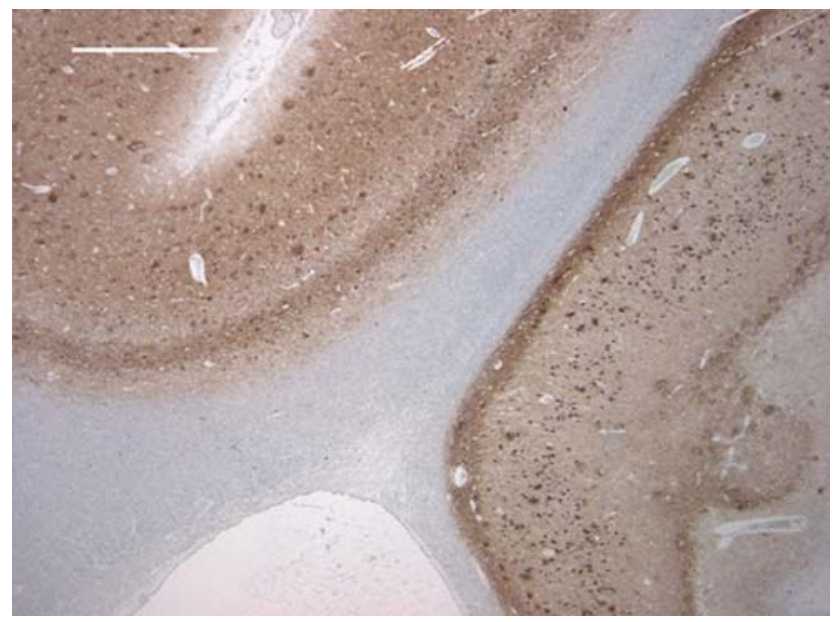

Fig. 7 Alzheimer's disease patient without aphasia. This section, including the depth of the collateral sulcus (top left) and the CA1 sector of the hippocampus immunostained with AT8, shows abundant plaques and tangles, but complete absence of subpial, subependymal, or white matter tau deposits in astrocytes. Bar $1 \mathrm{~mm}$

stages of PPA and Possible PPA are fluent but the main distinguishing feature is the episodic memory loss in possible PPA. Semantic dementia patients remain fluent but maintain episodic memory while loosing semantic memory and comprehension. The speech at the last assessment was nonfluent in four patients with ATAC and still fluent in the patient without ATAC. Aphasia was more prominent in all the possible PPA patients than those diagnosed as AD clinically.

The focal accentuation of the atrophy and asymmetry observed in imaging in half of the cases at presentation was much less prevalent at the time of autopsy, once the disease had run its course, a pattern commonly seen in FTLD. Interestingly, the patient without ATAC also showed left predominant atrophy.

Our second finding concerns the presence of ATAC in the majority of possible PPA cases. The constituent thorny astrocytes are a subtype of glial fibrillary tangles (GFT), argyrophilic masses containing deposits of abnormal tau proteins located in the cytoplasm of glial cells [37]. Electron microscopy typically shows bundles of $15 \mathrm{~nm}$ straight tubules [22]. All forms lack tau isoforms corresponding to the expression of alternatively spliced exons 2 and 3 [55], but otherwise are labeled with all tau antibodies. In contrast to neurofibrillary tangles, GFT are poorly labeled by antibodies to ubiquitin, and not at all by antibodies to apolipoprotein E, alpha-1-antichymotrypsin, and heparan sulfate [22]. Oligodendrocytes present a single form, named coiled 
bodies, whose processes appear as argyrophilic threads in the white matter [4], whereas at least three varieties can be identified in astrocytes. Two of these appear in protoplasmic astrocytes, identified by their CD44 positive, GFAP negative immunohistochemical profiles, are predominantly located in the cerebral cortex and subcortical grey masses rather than the white matter, and involve the processes rather than the cell body. Tufted astrocytes are characterized by fine process radiating from the inconspicuous cell body, whereas glial plaques consist of a crown of processes devoid of an obvious connection to a cell body. The other variety, named thorny astrocytes or thorn-shaped astrocytes, show a conspicuous GFAP positive cytoplasm drawn into funnel-shaped thick process, reaching as end feet perivascular subpial and subependymal linings [69].

In terms of the significance of GFT, coiled bodies are present in many taupathies, including dementia with argyrophilic grains [4, 25, 41], PSP [68], CBD [63], and subacute sclerosing panencephalitis [24]. Tufted astrocytes are considered pathognomonic of PSP [19, 67], and glial plaques are characteristic of CBD [8, 37, 38, 2],

The nosological significance of thorny astrocytes is less clear. Although initially described in small numbers in a limited area in patients with several diseases characterized by neuronal tau deposits [26], tau + thorny astrocytes in subpial and subependymal regions are common in the medial temporal lobe of elderly subjects, albiet only a small subset demonstrate argyrophilia. There is no correlation between the abundance of thorny astrocytes and neurofibrillary tangle stage, amyloid deposition, or argyrophilic grain disease severity. The presence of ApoE $\varepsilon 4$ allele is not associated with the presence of thorny astrocytes [58]. On the other hand, abundant ATAs have been restricted to postencephalitic parkinsonism of von-Economo type [23] and three forms of frontotemporal degeneration, FTLD with Pick bodies, PSP, and diffuse neurofibrillary tangles with calcification. In FTLD with Pick bodies they were described in the subcortical white matter of atrophic middle and inferior temporal gyri [70], or in affected cortical areas $[28,71]$. In PSP thorny astrocytes appear in areas with relatively minor neuronal loss including the striatum, thalamus, superior colliculus and frontal and motor cortex, but not in the white matter $[54,69]$. In dementia with diffuse neurofibrillary tangles with calcification, which can be considered a subset of neurofibrillary tangle dementia [43], thorny astrocytes form subcortical and cortical clusters, similar to our ATAC [18]. However the stated coincidence of the distribution of thorn-shaped astrocytes and gliosis [18] is at odds with our observations on ATAC.

Finally, two cases of PPA in which astrocytic tau deposits constituted the dominant pathology have been reported. The patient described by Molina et al. [49] was an 80-yearold woman with PPA who in a left temporal lobe biopsy showed astrocytes stained by Gallyas and containing tau and ubiquitin positive inclusions located in cortex and white matter, predominantly at the grey-white junction, as in our cases. In addition, coiled bodies were present. There was no hint of Alzheimer disease, nor were any tau deposits found in neurons other than rare diffuse perikaryal staining. Ballooned neurons and superficial spongiosis were not present. The second case, reported by Wakabayashi et al. [64] in a 72-year-old man with a 6-year typical aphemic history, showed tufted astrocytes in layers II-V in the lower third of the left primary motor area, and to a lesser degree in perirolandic cortex bilaterally. The astrocytic inclusions were stained by phosphorylation dependent (AT8) and independent (tau-2) antibodies, but not by antibodies to alpha synuclein, or ubiquitin. There were numerous oligodendroglial inclusions (coiled bodies) with the same immunoreactive pattern in deep cortical layers and white matter. Superficial linear spongiosis was observed in the affected regions. Western blot analysis of insoluble tau proteins after dephosphorylation was reported as demonstrating three major bands corresponding to $4 \mathrm{R}$ and $3 \mathrm{R}$ isoforms, with a preponderance of $4 \mathrm{R}$. Other findings included argyrophilic grains and a few neurofibrillary tangles in the hippocampus, but no neurofibrillary tangles in cortical or subcortical neurons. Thus, the authors did not consider that the patient's disease could be classified as PSP, even if the astrocytic inclusions were similar to those seen in this disease [64]. Abundant cortical tufted astrocytes are a characteristic feature in PSP cases presenting with PNFA [3, 27, 48].

It should be mentioned that mutations in exons 1 and 10 and the intron following exon 10 of the gene MAPT are associated with robust astrocytic and oligodendroglial, in addition to neuronal, tau deposition [11].

Argyrophilic thorny astrocyte cluster represents a lesion not fully characterized in the literature. The basic process of deposition of tau in GFAP immunoreactive fibrous astrocytes is one shared with aging, but in contrast to the periventricular, subependymal, and subpial location of the thorny astrocytes described in normal aged subjects [58], ATAC are located in the cerebral cortex and subcortical white matter, are not restricted to the medial temporal lobe but involve the frontal, temporal, and parietal lobes, and display intense argyrophilia. Similar points of distinction in relation to normal aging were advanced in the report of the clusters of GFTs associated with diffuse neurofibrillary tangles with calcification [18], lesions closely related to ATAC. More research will be necessary to establish whether ATAC represent an intensification of the astrocytic changes seen in aging, or else a different phenomenon, possibly related to FTLD pathologies [51] This distinction is well known in neuronal tau deposits, where neurofibrillary tangles in $\mathrm{AD}$ replicate the biochemical composition and 
extend the anatomical distribution of those in normal aged brains, whereas neurofibrillary tangles in CBD or progressive supranuclear palsy do neither [6]. Other issue for future research is the range of conditions in which ATAC appear. Our preliminary observations indicate that ATAC are not found in FTLD-U with either PPA or behavioral presentations.

We hypothesize that ATAC represent a marker of a process, co-exiting but probably independent of $\mathrm{AD}$, and responsible for the prominent focal clinical manifestations. The argument for the independence of ATAC and AD is grounded on cases in which subcortical thorny astrocytes are either the only reported pathology [49] or accompany FTLD with Pick bodies rather than AD [70]. In addition, the brains reported here showed no topographic relationship between the severity of plaques and tangles on the one hand and ATAC on the other. ATAC could be the result of pathogenetic processes related to those subjacent to FTLD. Alternatively, they could be secondary to alterations in the blood-brain barrier, a possibility raised in relation to the thorny astrocytes of normal aged brains [58], and compatible with ATAC's patchy, nonsystematic distribution. Although our findings are consistent with either hypothesis, it should be noted that a related lesion, tufted astrocytes in PSP, are considered a degenerative rather than reactive process because their distribution is unrelated to reactive astrocytes in the vicinity of infarcts or neurofibrillary tangles [62]. The consistent development in aged baboons, but not other primate species, of tau + thorny astrocytes in a stereotyped distribution suggests that genetic variations contribute to the formation of ATAC. An important focus for future research is the relationship between the marker (ATAC) and the clinical manifestation (aphasia).

Acknowledgments We thank Mervin Blair, Cecil Marcinski, Wilda Davidson and Pat McCabe for their assistance in testing and longitudinally following these patients.

\section{References}

1. Appell J, Kertesz A, Fisman M (1982) A study of language functioning in Alzheimer patients. Brain Lang 17:73-91

2. Bergeron C, Pollanen MS, Weyer L, Lang AE (1997) Cortical degeneration in progressive supranuclear palsy. A comparison with cortical-basal ganglionic degeneration. J Neuropathol Exp Neurol 56:726-734

3. Boeve B, Dickson D, Duffy J, Bartleson J, Trenerry M, Petersen R (2003) Progressive nonfluent aphasia and subsequent aphasic dementia associated with atypical progressive supranuclear palsy pathology. Eur Neurol 49:72-78

4. Braak H, Braak E (1989) Cortical and subcortical argyrophilic grains characterize a disease associated with adult onset dementia. Neuropathol Appl Neurobiol 15:13-26

5. Cairns NJ, Bigio EH, Mackenzie IR, Neumann M, Lee VM, Hatanpaa KJ, White CL III, Schneider JA, Grinberg LT, Halliday G, Duyckaerts C, Lowe JS, Holm IE, Tolnay M, Okamoto K, Yokoo
H, Murayama S, Woulfe J, Munoz DG, Dickson DW, Ince PG, Trojanowski JQ, Mann DM (2007) Neuropathologic diagnostic and nosologic criteria for frontotemporal lobar degeneration: consensus of the Consortium for Frontotemporal Lobar Degeneration. Acta Neuropathol (Berl) 114:5-22

6. Delacourte A, Sergeant N, Wattez A, Robitaille I (1998) The biochemistry of the cytoskeleton in Pick complex. In: Kertesz A, Munoz DG (eds) Pick's disease and Pick complex. Wiley-Liss, New York, pp 243-258

7. Deruaz JP, Assal G, Peter-Favre C (1993) Un cas clinicopathologique d'aphasie progressive. Rev Neurol (Paris) 149:186-191

8. Feany MB, Dickson DW (1995) Widespread cytoskeletal pathology characterizes corticobasal degeneration. Am J Pathol 146:1388-1396

9. Ferrer I, Hernandez I, Boada M, Llorente A, Rey MJ, Cardozo A, Ezquerra M, Puig B (2003) Primary progressive aphasia as the initial manifestation of corticobasal degeneration and unusual tauopathies. Acta Neuropathol (Berl) 106:419-435

10. Galton CJ, Patterson K, Xuereb JH, Hodges JR (2000) Atypical and typical presentations of Alzheimer's disease: a clinical, neuropsychological, neuroimaging and pathological study of 13 cases. Brain 123:484-498

11. Ghetti B, Hutton ML, Wszolek ZK (2003) Frontotemporal dementia and parkinsonism linked to chromosome 17 associated with tau gene mutations (FTDP-17T). In: Dickson DW (ed) Neurodegeneration: the molecular pathology of dementia of movement disorders, Basel

12. Goedert M, Jakes R, Vanmechelen E (1995) Monoclonal antibody AT8 recognises tau protein phosphorylated at both serine 202 and threonine 205. Neurosci Lett 189:167-169

13. Gorno-Tempini ML, Dronkers NF, Rankin KP, Ogar JM, Phengrasamy L, Rosen HJ, Johnson JK, Weiner MW, Miller BL (2004) Cognition and anatomy in three variants of primary progressive aphasia. Ann Neurol 55:335-346

14. Graff-Radford NR, Damasio AR, Hyman BT, Hart MN, Tranel D, Damasio H, Van Hoesen GW, Rezai K (1990) Progressive aphasia in a patient with Pick's disease: a neuropsychological, radiologic, and anatomic study. Neurology 40:620-626

15. Green J, Morris JC, Sandson J, McKeel DWJ, Miller JW (1990) Progressive aphasia: a precursor of global dementia? Neurology 40:423-429

16. Greene JD, Patterson K, Xuereb J, Hodges JR (1996) Alzheimer disease and nonfluent progressive aphasia. Arch Neurol 53:10721078

17. Harasty JA, Halliday GM, Xuereb J, Croot K, Bennett H, Hodges JR (2001) Cortical degeneration associated with phonologic and semantic language impairments in AD. Neurology 56:944-950

18. Hashimoto N, Takeuchi T, Ishihara R, Ukai K, Kobayashi H, Iwata H, Iwai K, Mizuno Y, Yamaguchi H, Shibayama H (2003) Glial fibrillary tangles in diffuse neurofibrillary tangles with calcification. Acta Neuropathol (Berl) 106:150-156

19. Hauw JJ, Verny M, Delaere P, Cervera P, He Y, Duyckaerts C (1990) Constant neurofibrillary changes in the neocortex in progressive supranuclear palsy. Basic differences with Alzheimer's disease and aging. Neurosci Lett 119:182-186

20. Holland AL, McBurney DH, Moossy J, Reinmuth OM (1985) The dissolution of language in Pick's disease with neurofibrillary tangles: a case study. Brain Lang 24:36-58

21. Hyman BT, Trojanowski JQ (1997) Consensus recommendations for the postmortem diagnosis of Alzheimer disease from the National Institute on Aging and the Reagan Institute Working Group on diagnostic criteria for the neuropathological assessment of Alzheimer disease. J Neuropathol Exp Neurol 56:1095-1097

22. Ikeda K, Akiyama H, Arai T, Nishimura T (1998) Glial tau pathology in neurodegenerative diseases: their nature and comparison with neuronal tangles. Neurobiol Aging 19:S85-S91 
23. Ikeda K, Akiyama H,.Kondo H (1993) Anti-tau-positive glial fibrillary tangles in the brain of postencephalitic parkinsonism of Economo type. Neurosci Lett 162:176-178

24. Ikeda K, Akiyama H, Kondo H, Arai T, Arai N,Yagishita S (1995) Numerous glial fibrillary tangles in oligodendroglia in cases of subacute sclerosing panencephalitis with neurofibrillary tangles. Neurosci Lett 194:133-135

25. Ikeda K, Akiyama H, Kondo H, Haga C (1995) A study of dementia with argyrophilic grains. Possible cytoskeletal abnormality in dendrospinal portion of neurons and oligodendroglia. Acta Neuropathol (Berl) 89:409-414

26. Ikeda K, Akiyama H, Kondo H, Haga C, Tanno E, Tokuda T, Ikeda S (1995) Thorn-shaped astrocytes: possibly secondarily induced tau-positive glial fibrillary tangles. Acta Neuropathol (Berl) 90:620-625

27. Iwasaki Y, Yoshida M, Hattori M, Goto A, Aiba I, Hashizume Y, Sobue G (2004) Distribution of tuft-shaped astrocytes in the cerebral cortex in progressive supranuclear palsy. Acta Neuropathol (Berl) 108:399-405

28. Iwatsubo T, Hasegawa M, Ihara Y (1994) Neuronal and glial taupositive inclusions in diverse neurologic diseases share common phosphorylation characteristics. Acta Neuropathol (Berl) 88:129136

29. Kertesz A (1980) Western aphasia battery. San Antonio, TX, The Psychological Corporation. (GENERIC) Ref Type: Generic

30. Kertesz A, Davidson W, McCabe P, Takagi K, Munoz D (2003) Primary progressive aphasia: diagnosis, varieties, evolution. J Int Neuropsychol Soc 9:710-719

31. Kertesz A, Hudson L, Mackenzie IR, Munoz DG (1994) The pathology and nosology of primary progressive aphasia. Neurology 44:2065-2072

32. Kertesz A, McMonagle P, Blair M, Davidson W, Munoz DG (2005) The evolution and pathology of frontotemporal dementia. Brain 128:1996-2005

33. Kertesz A, Munoz DG (2002) Primary progressive aphasia: a review of the neurobiology of a common presentation of Pick complex. Am J Alzheimers Dis Other Demen 17:30-36

34. Kinoshita A, Tomimoto H, Tachibana N, Suenaga T, Kawamata T, Kimura T, Akiguchi I, Kimura J (1996) A case of primary progressive aphasia with abnormally ubiquitinated neurites in the cerebral cortex. Acta Neuropathol (Berl) 92:520-524

35. Kirshner HS, Tanridag O, Thurman L, Whetsell WO Jr (1987) Progressive aphasia without dementia: two cases with focal spongiform degeneration. Ann Neurol 22:527-532

36. Knibb JA, Xuereb JH, Patterson K, Hodges JR (2006) Clinical and pathological characterization of progressive aphasia. Ann Neurol 59:156-165

37. Komori T (1999) Tau-positive glial inclusions in progressive supranuclear palsy, corticobasal degeneration and Pick's disease. Brain Pathol 9:663-679

38. Komori T, Arai N, Oda M, Nakayama H, Mori H, Yagishita S, Takahashi T, Amano N, Murayama S, Murakami S, Shibata N, Kobayashi M, Sasaki S, Iwata M (1998) Astrocytic plaques and tufts of abnormal fibers do not coexist in corticobasal degeneration and progressive supranuclear palsy. Acta Neuropathol (Berl) 96:401-408

39. Lippa CF, Cohen R, Smith TW, Drachman DA (1991) Primary progressive aphasia with focal neuronal achromasia. Neurology 41:882-886

40. Manlow A, Munoz DG (1992) A non-toxic method for the demonstration of gliosis. J Neuropathol Exp Neurol 51:298-302

41. Martinez-Lage P, Munoz DG (1997) Prevalence and disease associations of argyrophilic grains of Braak. J Neuropathol Exp Neurol 56:157-164

42. McKhann G, Drachman D, Folstein M, Katzman R, Price D, Stadlan EM (1984) Clinical diagnosis of Alzheimer's disease: report of the NINCDS-ADRDA Work Group under the auspices of Department of Health and Human Services Task Force on Alzheimer's Disease. Neurology 34:939-944

43. McKhann GM, Albert MS, Grossman M, Miller B, Dickson D, Trojanowski JQ (2001) Clinical and pathological diagnosis of frontotemporal dementia: report of the Work Group on Frontotemporal Dementia and Pick's Disease. Arch Neurol 58:1803-1809

44. Mehler MF, Horoupian DS, Davies P, Dickson DW (1987) Reduced somatostatin-like immunoreactivity in cerebral cortex in nonfamilial dysphasic dementia. Neurology 37:1448-1453

45. Mesulam MM (1982) Slowly progressive aphasia without generalized dementia. Ann Neurol 11:592-598

46. Mesulam MM (2001) Primary progressive aphasia. Ann Neurol 49:425-432

47. Mirra SS, Heyman A, McKeel D, Sumi SM, Crain BJ, Brownlee LM, Vogel FS, Hughes JP, van-Belle G, Berg L (1991) The Consortium to Establish a Registry for Alzheimer's Disease (CERAD). Part II. Standardization of the neuropathologic assessment of Alzheimer's disease. Neurology 41:479-486

48. Mochizuki A, Ueda Y, Komatsuzaki Y, Tsuchiya K, Arai T, Shoji S (2003) Progressive supranuclear palsy presenting with primary progressive aphasia-clinicopathological report of an autopsy case. Acta Neuropathol (Berl) 105:610-614

49. Molina JA, Probst A, Villanueva C, Jimenez-Jimenez FJ, Madero S, Torres N, Bermejo F (1998) Primary progressive aphasia with glial cytoplasmic inclusions. Eur Neurol 40:71-77

50. Munoz DG (1999) Stains for the differential diagnosis of degenerative dementias. Biotech Histochem 74:311-320

51. Munoz DG, Dickson DW, Bergeron C, Mackenzie IR, Delacourte A, Zhukareva V (2003) The neuropathology and biochemistry of frontotemporal dementia. Ann Neurol 54:S24-S28

52. Munoz DG, Kertesz A (2001) The neuropathology of primary progressive aphasia. Neurology 56(Suppl 3):A178 (Abstract)

53. Munoz DG, Woulfe J, Rabano A, Gerrero C, Kertesz A (2003) Glial Tau pathology as the specific structural correlate of focal presentation of Alzheimer's disease. Neurology 60(Suppl 1):A312 (Abstract)

54. Nishimura M, Namba Y, Ikeda K, Oda M (1992) Glial fibrillary tangles with straight tubules in the brains of patients with progressive supranuclear palsy. Neurosci Lett 143:35-38

55. Nishimura T, Ikeda K, Akiyama $\mathrm{H}$, Arai T, Kondo H, Okochi M, Furiya Y, Mori H, Oda T, Kato M, Iseki E (1997) Glial tau-positive structures lack the sequence encoded by exon 3 of the tau protein gene. Neurosci Lett 224:169-172

56. Pogacar S, Williams RS (1984) Alzheimer's disease presenting as slowly progressive aphasia. R I Med J 67:181-185

57. Scheltens P, Ravid R, Kamphorst W (1994) Pathologic findings in a case of primary progressive aphasia. Neurology 44:279-282

58. Schultz C, Ghebremedhin E, Del Tredici K, Rub U, Braak H (2004) High prevalence of thorn-shaped astrocytes in the aged human medial temporal lobe. Neurobiol Aging 25:397-405

59. Schwarz M, De BR, Poeck K, Weis J (1998) A case of primary progressive aphasia. A 14-year follow-up study with neuropathological findings. Brain 121:115-126

60. Shi J, Shaw CL, Du PD, Richardson AM, Bailey KL, Julien C, Stopford C, Thompson J, Varma A, Craufurd D, Tian J, PickeringBrown S, Neary D, Snowden JS, Mann DM (2005) Histopathological changes underlying frontotemporal lobar degeneration with clinicopathological correlation. Acta Neuropathol (Berl) 110:501-512

61. Snowden JS, Neary D, Mann DM, Goulding PJ, Testa HJ (1992) Progressive language disorder due to lobar atrophy. Ann Neurol 31:174-183

62. Togo T, Dickson DW (2002) Tau accumulation in astrocytes in progressive supranuclear palsy is a degenerative rather than a reactive process. Acta Neuropathol (Berl) 104:398-402

63. Wakabayashi K, Oyanagi K, Makifuchi T, Ikuta F, Homma A, Homma Y, Horikawa Y,Tokiguchi S (1994) Corticobasal degeneration: 
etiopathological significance of the cytoskeletal alterations. Acta Neuropathol (Berl) 87:545-553

64. Wakabayashi K, Shibasaki Y, Hasegawa M, Horikawa Y, Soma Y, Hayashi S, Morita T, Iwatsubo T, Takahashi H (2000) Primary progressive aphasia with focal glial tauopathy. Neuropathol Appl Neurobiol 26:477-481

65. Watanabe N, Takio K, Hasegawa M, Arai T, Titani K, Ihara Y (1992) Tau 2: a probe for a Ser conformation in the amino terminus of tau. J Neurochem 58:960-966

66. Wechsler AF, Verity MA, Rosenschein S, Fried I, Scheibel AB (1982) Pick's disease. A clinical, computed tomographic, and histologic study with golgi impregnation observations. Arch Neurol 39:287-290

67. Yamada T, Calne DB, Akiyama H, McGeer EG, McGeer PL (1993) Further observations on Tau-positive glia in the brains with progressive supranuclear palsy. Acta Neuropathol (Berl) 85:308-315
68. Yamada T, McGeer PL (1990) Oligodendroglial microtubular masses: an abnormality observed in some human neurodegenerative diseases. Neurosci Lett 120:163-166

69. Yamada T, McGeer PL, McGeer EG (1992) Appearance of paired nucleated, Tau-positive glia in patients with progressive supranuclear palsy brain tissue. Neurosci Lett 135:99-102

70. Yamazaki M, Nakano I, Imazu O, Terashi A (1995) Paired helical filaments and straight tubules in astrocytes: an electron microscopic study in dementia of the Alzheimer type. Acta Neuropathol (Berl) 90:31-36

71. Yasuhara O, Matsuo A, Tooyama I, Kimura H, McGeer EG, McGeer PL (1995) Pick's disease immunohistochemistry: new alterations and Alzheimer's disease comparisons. Acta Neuropathol (Berl) 89:322-330 